\title{
MINIMUM THICKNESS AND COLLAPSE CONDITIONS OF THE IRREGULAR MASONRY ARCH SUBJECT TO ITS OWN WEIGTH
}

\author{
N. CAVALAGLI* , V. GUSELLA AND R. LIBEROTTI \\ Department of Civil and Environmental Engineering \\ Univrsity of Perugia \\ Via G. Duranti 93, 06125, Perugia, Italy \\ e-mail: \{nicola.cavalagli,vittorio.gusella,riccardo.liberotti\}@unipg.it (*corresponding author)
}

Keywords: Masonry arch, Irregular geometry, Uncertainties, Minimum thickness

\begin{abstract}
The present paper aims at assessing the shape irregularities influence on the collapse conditions of masonry arches subjected to vertical point force. Surveys campaigns on cultural Heritage buildings often reveal masonry elements with irregular shapes. In order to accurately predict their actual behavior the presence of such features cannot be neglected. The reasons for these non-canonical shapes are manifold e.g. the evolution of degradation processes and the constructive laws. As a first analysis attempt, the geometrical uncertainties tied to the building work criteria are taken into account. A random parametric model is proposed to generate two-dimensional geometry of irregular polycentric arches. Monte Carlo simulations allows estimating the probabilistic density function of the collapse load multiplier evaluated by means of the implemented limit analysis method. The outcomes referred to the masonry arch with nominal geometry are compared to those obtained for the random polycentric arch, highlighting the extent of the load-bearing capacity reduction due to presence of such shape irregularities.
\end{abstract}

\section{INTRODUCTION}

It is well known that the level of knowledge and the in-depth investigations of ancient masonry buildings are the mandatory conditions on which reliable structural safety assessments must be based. Those evaluations derive from an articulated and rather complex process including, in general, historical investigations gathered by direct surveys of the building, also by means of experimental-test campaigns [[1], [2], [3], [4]]. In order to produce vulnerability evaluations on the safe side, the challenge to represent a correct interpretation of the seismic behaviour of historical constructions and in particular masonry bridges (e.g. see Figure 1), in their current state, becomes an essential prerequisite especially in presence of peculiarities, as material degradation and structural damages. In these sense, masonry arches and vaults play a significant role due to their extensive use not only in historical bridges, but also in civil and monumental structures in general. For this reasons, the research activities were continuously devoted to increase the knowledge of their structural behaviour until today [[5], [6], [7], [8], [9], [10], [11], [12], [13]]. 


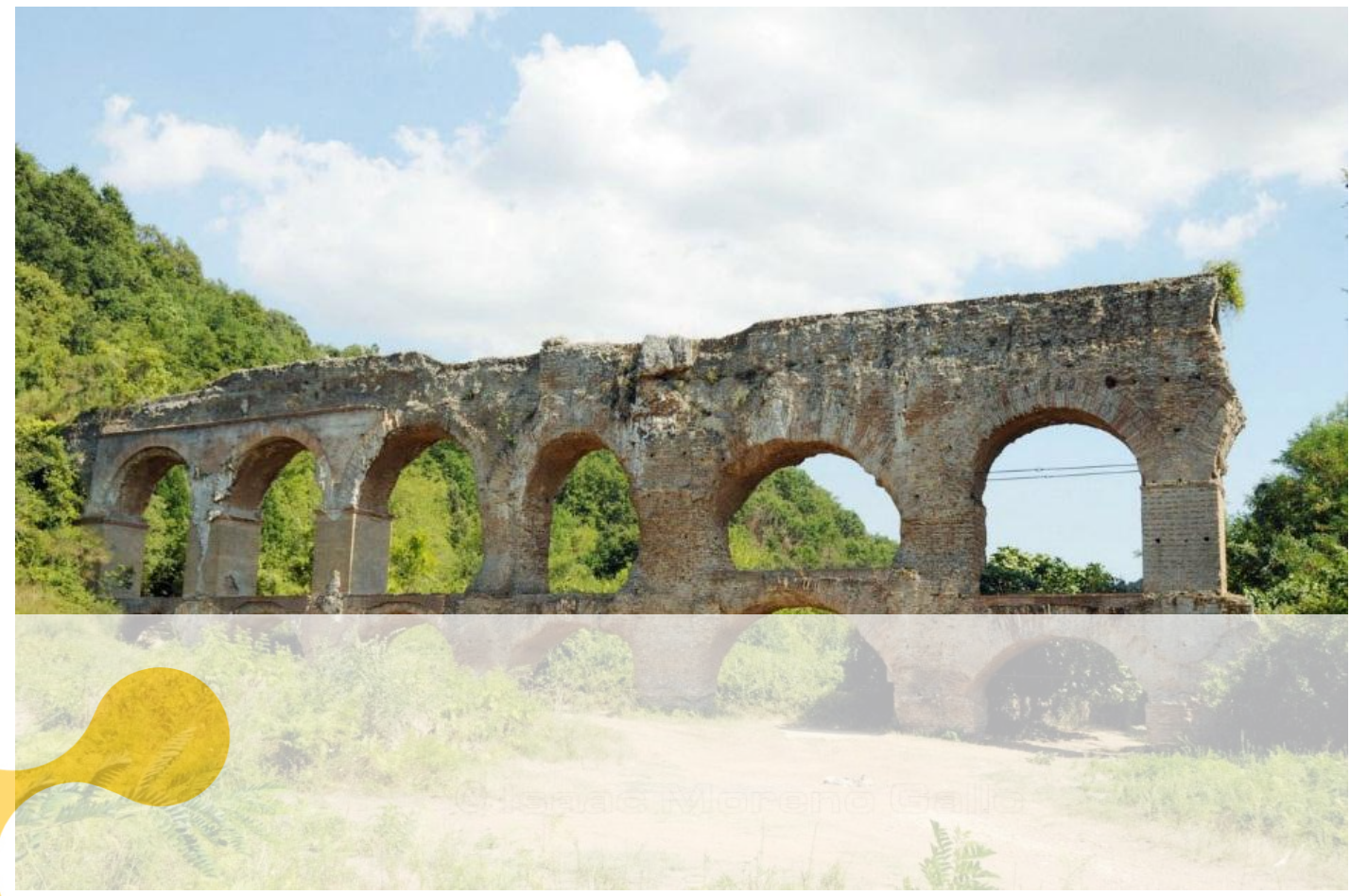

Figure 1: Ponte della Mola. Roman aqueduct, sited near Rome, between Tivoli and Palestrina, built from 272 and

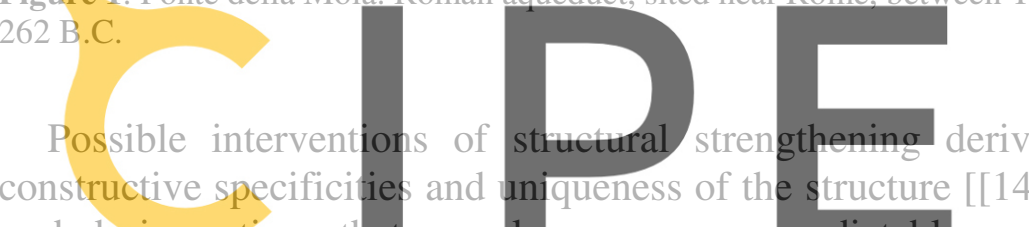

such design actions, that as we know are never predictable
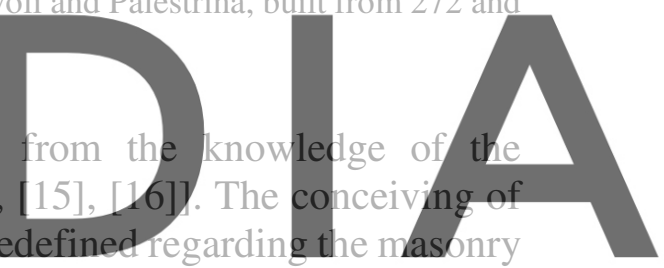

arched structures the plurality of monuments and infrastructures in terms of construction

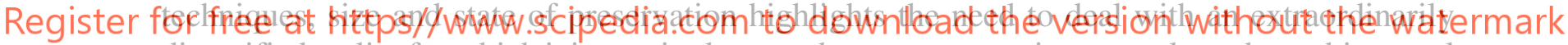
diversified reality for which it is required to pay the utmost attention not only to the architectural appearance but also to the shape of the basic construction elements e.g. the correspondence between stone blocks and identification of non-original parts [[17], [18]]. Likewise, the phenomena of material degradation, biological patinas, detachment of walls, surface weathering, leaks and erosion must be ascribed as sources of geometrical uncertainties and need to be documented by introducing correlations between causes and effects. In this perspective, in addition, the scientific literature proved to be lacking except from specific papers where the masonry arches have been characterized according to their geometric features [[19], [20]], or where the damage pattern have been analyzed with deterministic or parametric variables [[21], [22]].

In previous contributions proposed by the Authors, the influence of geometrical uncertainties on the structural behaviour of masonry elements has been studied engaging the arches with horizontal actions through static and dynamic approaches [[23], [24], [25]]. Contextually the uncertainties at the voussoirs scale have been introduced, taking into account the randomness of the parameters describing the masonry texture. Those first results proved that for a certain level of irregularities the drop of strength cannot be neglected, as just asserted by several authors in the interpretation of experimental tests. Differently in this paper, the item of the shape 
irregularities is treated at a macro scale, defining them as the geometrical variance from the nominal arch, and ascribed to the aprioristic inaccuracies connected to the ancient constructive criteria [26]. This choice founds argumentative roots in the observation of rather articulated historical buildings often composed by masonry clusters combining different floor heights, where some load bearing walls or vaults rest on the underlying arches.

\section{DEFINITION OF A MASONRY ARCH WITH IRREGULAR SHAPE}

Over architectural and infrastructural heritage of Europe and more, the masonry arch represents the most used constructive system. Its building work requires the installation of temporary scaffoldings and frameworks in order to prop up the structure until it turns into a self-supporting system. The design of such wooden frames, known as centrings, envisages the union of a series of different arc sectors with a view to outline the intrados corresponding with the designed arch. Is thought that this criterion may lead to constructive imprecisions and, for this, within the present contribution this topic is highlighted as a possible source of irregularities in the final shape of the arches. In addition it should be noted that, when composed by several parts for the construction of large structures, the centrings were always built up in situ, resulting in unavoidable geometrical distortions from the nominal geometry, provided by wooden imperfections and manpower activities. The structural influence of such aspect has been addressed within previous contributions [[27], [28]]. The number of the arc sectors which constitute a centring are related to the vernacular building work traditions, the historical period and, above all, of the geometry and dimension supposed for the masonry arch.

In this study, an arch for moderately sized string

final shape of the arch, sectors affected by therefore composed
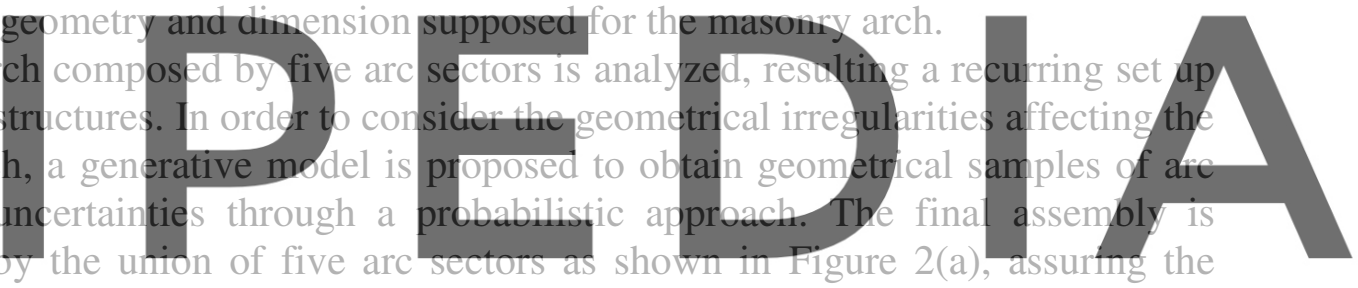
continuity of the tangent function along the development of the arch. The result is a procedure

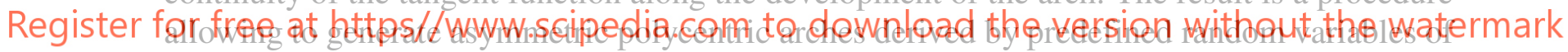
specific geometrical parameters $\tilde{\alpha}_{S}$ and $\tilde{r}_{S}$, identifying the angle of embrace and the radius of each arc sector. The obtained line of axis of the polycentric arch is finally scaled in order to have a span $l$ equal to the nominal span value (Figure 2(b)).

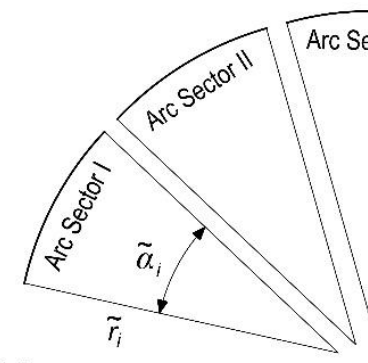

(a)

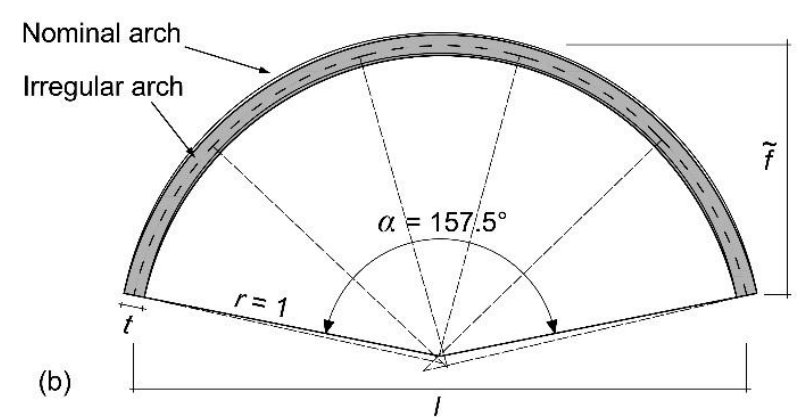

Figure 2: (a) Graphical construction of the arch irregular shape constituted by five random arc sectors. (b) Superimposition between the nominal and irregular arch. 
The nominal deterministic geometry analyzed in this work refers to a circular arch defined by the following parameters

$$
\left\{\begin{array}{c}
\alpha=157.5^{\circ} \\
r=1
\end{array}\right.
$$

being $\alpha$ the angle of embrace and $r$ the radius of the arch; the thickness $t$ is considered constant along all the development of the arch. Given the nominal geometry, the random variables $\tilde{\alpha}_{S}$ and $\tilde{r}_{S}$ are defined by the following relations

$$
\begin{aligned}
& \tilde{\alpha}_{S}=E\left[\tilde{\alpha}_{S}\right]+\varepsilon \alpha / N_{S} \cdot \tilde{p}_{\alpha_{S}}=\alpha / N_{S}+\varepsilon \alpha / N_{S} \cdot \tilde{p}_{\alpha_{S}} \\
& \tilde{r}_{S}=E\left[\tilde{r}_{S}\right]+\chi^{r} \cdot \tilde{p}_{r_{S}}=r+\chi^{r} \cdot \tilde{p}_{r_{S}}
\end{aligned}
$$

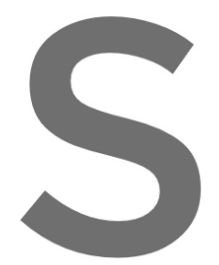

where $N_{S}$ represents the number of arc sectors (in this case $N_{S}=5$ will be considered), $\varepsilon$ and $\chi$ are defined as geometrical uncertainty coefficients which independently scale the uniform probability density functions $\tilde{p}_{\alpha_{s}}$ and $\tilde{p}_{r_{s}}$ respectively (Figure 3). Finally, no variability is considered for the thickness $t$.

\section{Register for free at https//www.sêpedia.com to download the vërsion withoüt the watermark (a) $\quad \varepsilon \cdot \alpha / n \varepsilon \cdot \alpha / n$

Figure 3: Uniform probability density functions used for the description of the angle of embrace (a) and radius (b) random parameters.

\section{MINIMUM THICKNESS OF THE IRREGULAR MASONRY ARCH}

\subsection{Collapse condition}

In the analysis of the minimum thickness of a masonry arch, the collapse condition, generally expressed in terms of collapse load and related mechanism, is strongly dependent on the structural shape and the distribution of the loads applied. It is well known that under its own weight, the collapse condition of a masonry arch is characterized by a symmetric five-hinges mechanism, with two extrados hinges at the imposts, one extrados hinge at the crown and two intrados hinges placed between the imposts and the crown, which position is function of the angle of embrace [29].

On the other hand, an irregular shape significantly affects the position of the hinges, which 
lead to the collapse. The introduction of irregularities causes the loss of symmetry both in geometry and in the distribution of the vertical loads. This condition determines an asymmetric collapse mechanism of four-hinges type, characterized by the occurrence of only one hinge at the imposts, alternatively at left or right on the basis of the thrust line shape.

\subsection{Work hypothesis and iterative procedure}

In this paper, the analysis of the minimum thickness of an arch with irregular shape is carried out through a limit analysis based procedure, aimed at evaluating the collapse load multiplier applied to an auxiliary loads distribution, which leads the structure to the collapse through a four-hinges mechanism. By progressively reducing the thickness of the arch, the collapse load multiplier goes to zero and the contribution of the auxiliary loads vanishes. The thickness associated to a null value of the load multiplier is taken as the minimum thickness of the masonry arch. In this study a distribution of inertial loads was considered as auxiliary load.

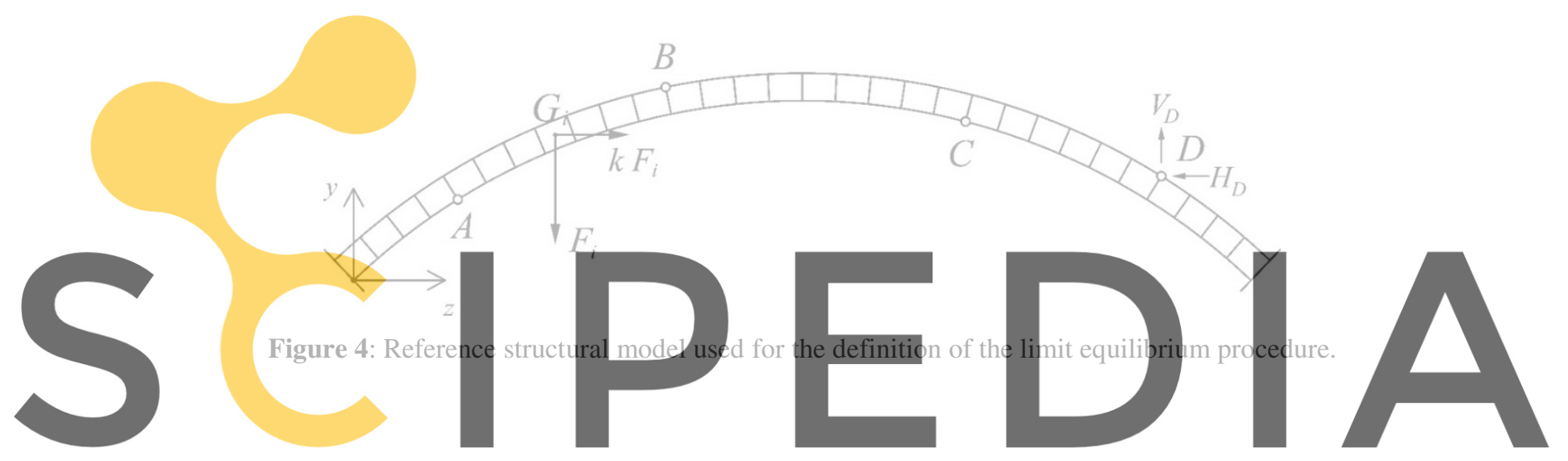

Let us consider a masonry arch consisting of $n$ voussoirs, on which is acting the self-weight

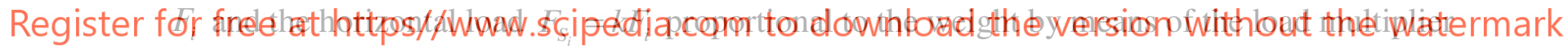

$k$. In the numerical procedure, the horizontal load is assumed directed from left to right, leading

the structure to the collapse with a four-hinges mechanism as indicated in Figure 4. The collapse condition, identified by the horizontal load multiplier $k$ and the corresponding hinges position associated to an admissible kinematic mechanism, is evaluated through an iterative procedure. A first attempt position for the collapse hinges $A, B, C, D$ is assigned, i.e. a collapse mechanism is assumed. Then, the equilibrium of moments around the three hinges $A, B$ and $C$ is imposed

$$
\left\{\begin{array}{l}
H_{D}\left(y_{A}-y_{D}\right)+V_{D}\left(z_{A}-z_{D}\right)-\sum_{i=1}^{n_{A D}} F_{i}\left(z_{A}-z_{G_{i}}\right)-k \cdot \sum_{i=1}^{n_{A D}} F_{i}\left(y_{A}-y_{G_{i}}\right)=0 \\
H_{D}\left(y_{B}-y_{D}\right)+V_{D}\left(z_{B}-z_{D}\right)-\sum_{i=1}^{n_{B D}} F_{i}\left(z_{B}-z_{G_{i}}\right)-k \cdot \sum_{i=1}^{n_{B D}} F_{i}\left(y_{B}-y_{G_{i}}\right)=0 \\
H_{D}\left(y_{C}-y_{D}\right)+V_{D}\left(z_{C}-z_{D}\right)-\sum_{i=1}^{n_{C D}} F_{i}\left(z_{C}-z_{G_{i}}\right)-k \cdot \sum_{i=1}^{n_{C D}} F_{i}\left(y_{C}-y_{G_{i}}\right)=0
\end{array}\right.
$$



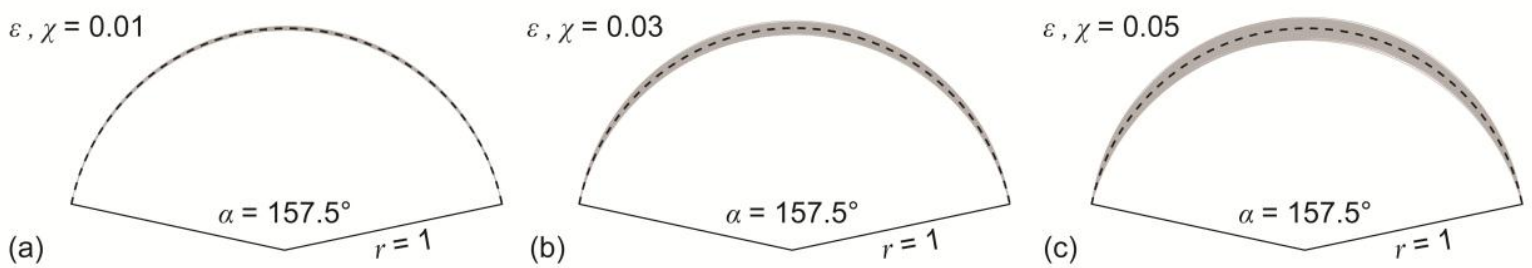

Figure 5: Population of the irregular arches (grey continuous line), generated through uncertainty parameters equal to 0.01 (a), 0.03 (b) and 0.05 (c), compared with the nominal arch (black dashed line).

where $n_{A D} n_{B D}, n_{C D}$, refer respectively to the number of voussoirs between the hinges $A, B, C$ and $D$. The system of Equations (e) is solved on the three unknowns $H_{D}, V_{D}$ and $k$. Finally, the thrust line is determined through the calculation of the joint-by-joint eccentricity $e_{j}$ of the normal force and assuring the satisfaction of the yielding criterion

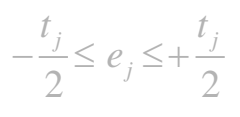

If the thrust line is not contained everywhere inside the boundaries of the arch geometry, Equation (3) is not somewhere verified. In this case a new trial configuration of hinges has to be considered and the equilibrium imposed again. The interested reader can see [23] for more

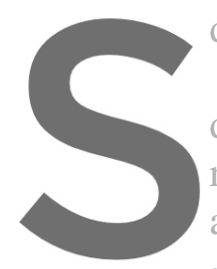
details.

As already discussed define in advance the we right side. In the application of the proposec $p$ applied independently multipliers, $k_{l}$ and $k_{r}$ respectively. The lower
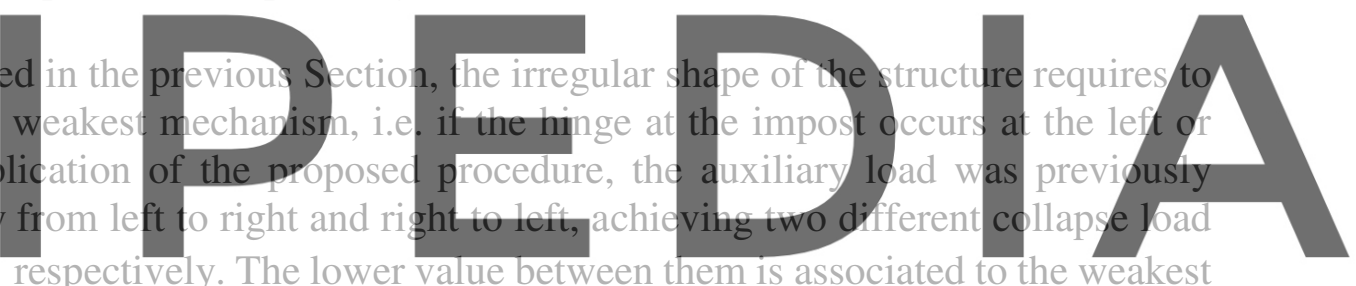

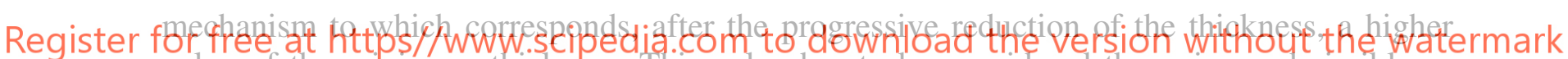
value of the minimum thickness. This value has to be considered the unique admissible as minimum thickness of the irregular arch, due to the occurrence of the collapse.

\subsection{Analysis of the results}

In this section, the results regarding the estimation of a probabilistic measure of the minimum thickness and the related collapse mechanism of masonry arches affected by three different levels of geometrical irregularities are shown. The analysis was carried out considering a nominal geometry defined by the properties indicated in Equations (1). A population of 1000 samples of random arches was generated according to the roles introduced in Section 2, for each value of the uncertainty coefficients $\varepsilon$ and $\chi$. In particular, uncertainty parameters equal to $0.01,0.03$ and 0.05 were considered.

In Figure 5 the line of axis of the generated irregular arches (grey continuous lines) is superimposed to the line of axis of the nominal arch (black dashed line), in order to give an idea of how the uncertainty parameters can affect the structural geometry. A distance measure between the nominal and the generic irregular arch is proposed by introducing the random parameter $\tilde{\rho}(\xi)$ as follows 


$$
\tilde{\rho}(\xi)=\frac{\tilde{r}_{n}(\xi)-r}{r}
$$

where $\xi$ is the curvilinear abscissa of the of the nominal arch normalized with respect to its total length, $\tilde{r}_{n}(\xi)$ is the radial distance between the irregular arch and the center of the nominal arch and $r$ is the nominal radius. Figure 6 illustrates the plots of the obtained $\tilde{\rho}(\xi)$ functions at different levels of the uncertainty parameters.
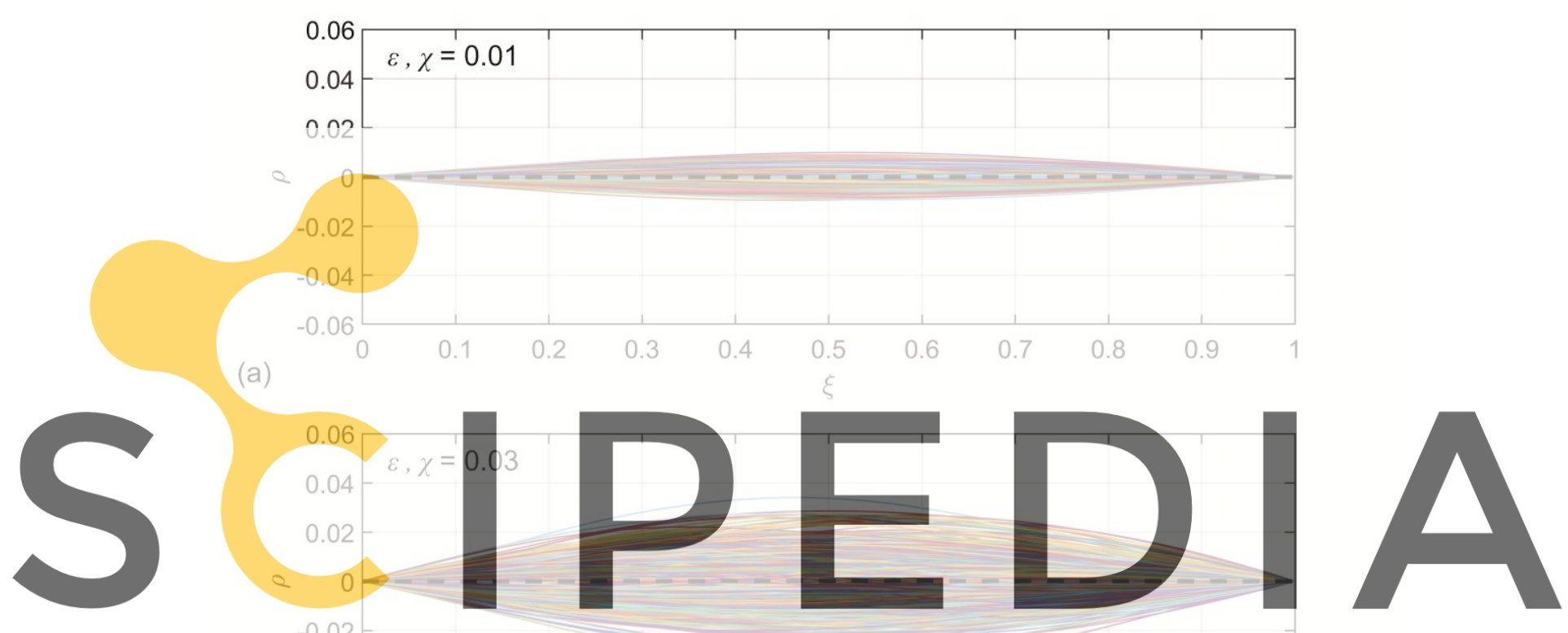

Register for free at.\$ttps//www.scipedia.com to download the version without the watermark

(b)
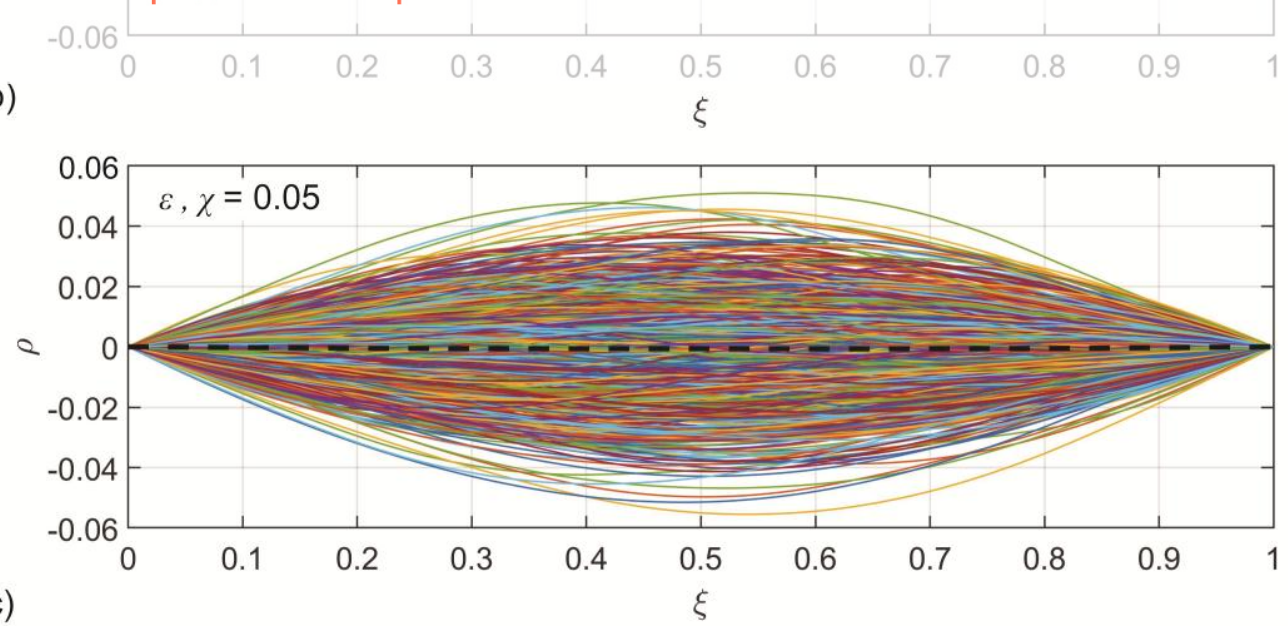

Figure 6: Plots of the radial distances between the irregular and the nominal arches with reference to uncertainty parameters equal to 0.01 (a), 0.03 (b) and 0.05 (c). 

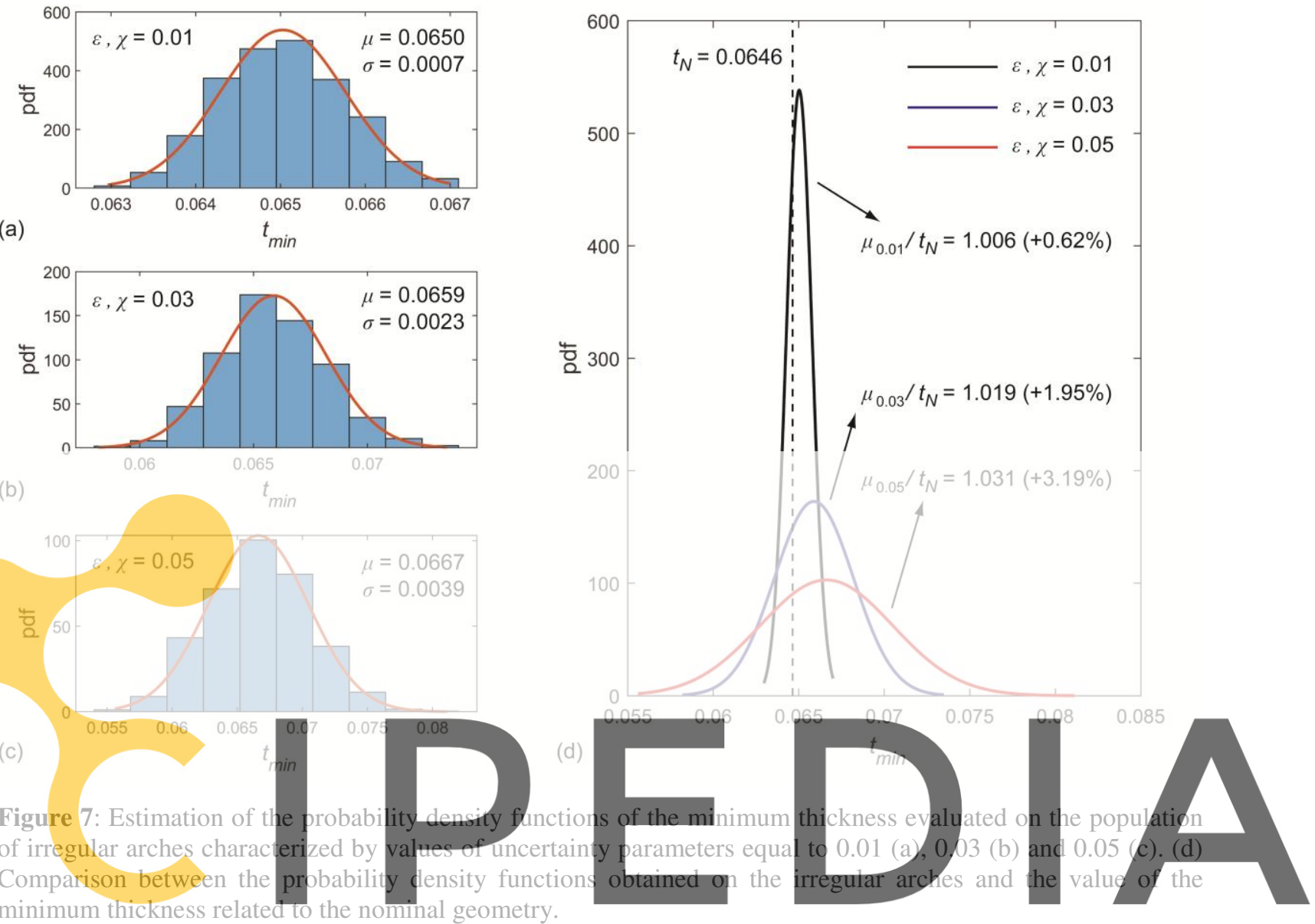

Register for free at https//www.scipedia.com to download the version without the watermark By analyzing the path of the $\rho(\xi)$ functions, it should be noted that in some cases the

irregular shape is characterized by values of the radial distance wherever positive or, alternatively, negative, resulting in an arch having a quasi-symmetric shape with a rise higher or lower with reference to the nominal rise. This aspect must be taken into account in the interpretation of the results expressed in terms of the minimum thickness. The presence of quasi-symmetric arches with a rise value lower than the nominal value determines the presence of minimum thicknesses lower than the minimum thickness related to the nominal arch, $t_{N}=0.0646$. Figure 7 shows the results obtained in terms of the random values of the minimum thickness $\tilde{t}_{\min }$ at different values of the uncertainty parameters. In particular, an estimation of the probability density function was obtained for $\varepsilon$ and $\chi$ equal to $0.01,0.03$ and 0.05 (Figure 7(a)-(c)). The mean $\mu$ and standard deviation $\sigma$ values reflect a slight increase of the minimum thickness, up to about $3 \%$, with the increase of the uncertainties and, at the same time, an increase of the values dispersion around the mean value. Figure 7(d) summarizes the comparison between the obtained results highlighting the different probabilistic properties of the irregular arches, with reference to the arch having nominal geometry. 
Table 1: Mean $(\mu)$ and standard deviation $(\sigma)$ values of the hinges $\left(\tilde{\beta}_{i}\right)$ probabilistic distributions identifying the collapse mechanism.

\begin{tabular}{ccccccc}
\hline & \multicolumn{2}{c}{$\tilde{\beta}_{1}$} & \multicolumn{2}{c}{$\tilde{\beta}_{2}$} & \multicolumn{2}{c}{$\tilde{\beta}_{3}$} \\
\hline & $\mu$ & $\sigma$ & $\mu$ & $\sigma$ & $\mu$ & $\sigma$ \\
\hline$\varepsilon, \chi=0.01$ & 0.7045 & 0.0068 & 1.5705 & 0.0068 & 2.4374 & 0.0068 \\
\hline$\varepsilon, \chi=0.03$ & 0.7017 & 0.0107 & 1.5709 & 0.0076 & 2.4398 & 0.0106 \\
\hline$\varepsilon, \chi=0.05$ & 0.6990 & 0.0162 & 1.5706 & 0.0088 & 2.4428 & 0.0163 \\
\hline
\end{tabular}

The irregular shape determines a variability of the results also in the collapse mechanism. As already stated in the previous Section, the symmetric five-hinges kinematic mechanism never occurs due to the asymmetry of the line of axis shape. The mechanism is now characterized by four hinges, among them only one occurs at the impost, either the left or the right with the same probability, i.e. $50 \%$. Regarding the probability distributions of the hinges position along the development of the arch expressed in terms of the angle $\tilde{\beta}_{i}$, in Table 1 the first statistical moments are reported for the different cases of uncertainty parameters considered. It should be noted that more spread results were obtained for the lateral hinges, $\tilde{\beta}_{1}$ and $\tilde{\beta}_{3}$, with respect to the crown hinge $\tilde{\beta}_{2}$. In particular, with the increase of the uncertainties, the mean values of $\tilde{\beta}$, and $\tilde{\beta}_{3}$ show a slight movement toward the left and right imposts, respectively, and an increase
of the standard deviation. The hinge at the crown presents more stable values in terms of both
mean and standard deviation. In Figure 8 the histograms of the randon variables $\tilde{\beta}_{i}$ are
illustrated for each values of the uncertainty parameters and their range of variability are highlighted with grey fills ( $\varepsilon, \chi=0.01$ dark grey, $\varepsilon, \chi=0.03$ grey, $\varepsilon, \chi=0.05$ light grey).

\section{CONCLUSIONS}

In this paper, the results of an investigation regarding the effect of the geometrical irregularities on the minimum thickness and the collapse mechanism of a masonry arch subject to its own weight have been presented.

For the definition of the irregular shape, a probabilistic generative procedure has been proposed by considering a polycentric arch constituted by five arc sectors described by two random parameters, the angle of embrace and the radius. For each type of random parameters, a unique uniform probability function has been considered. Three levels of uncertainties have been investigated: $1 \%, 3 \%$ and $5 \%$ of the nominal value of the considered parameter.

As a reference of nominal geometry, a circular arch of unit radius and angle of embrace of $157.5^{\circ}$ has been taken into account. All the samples of random arches generated by the procedure have been scaled in order to have the same span of the arch with nominal geometry. The minimum thickness has been studied as a random variable, obtained through a numerical procedure based on the limit equilibrium analysis. 


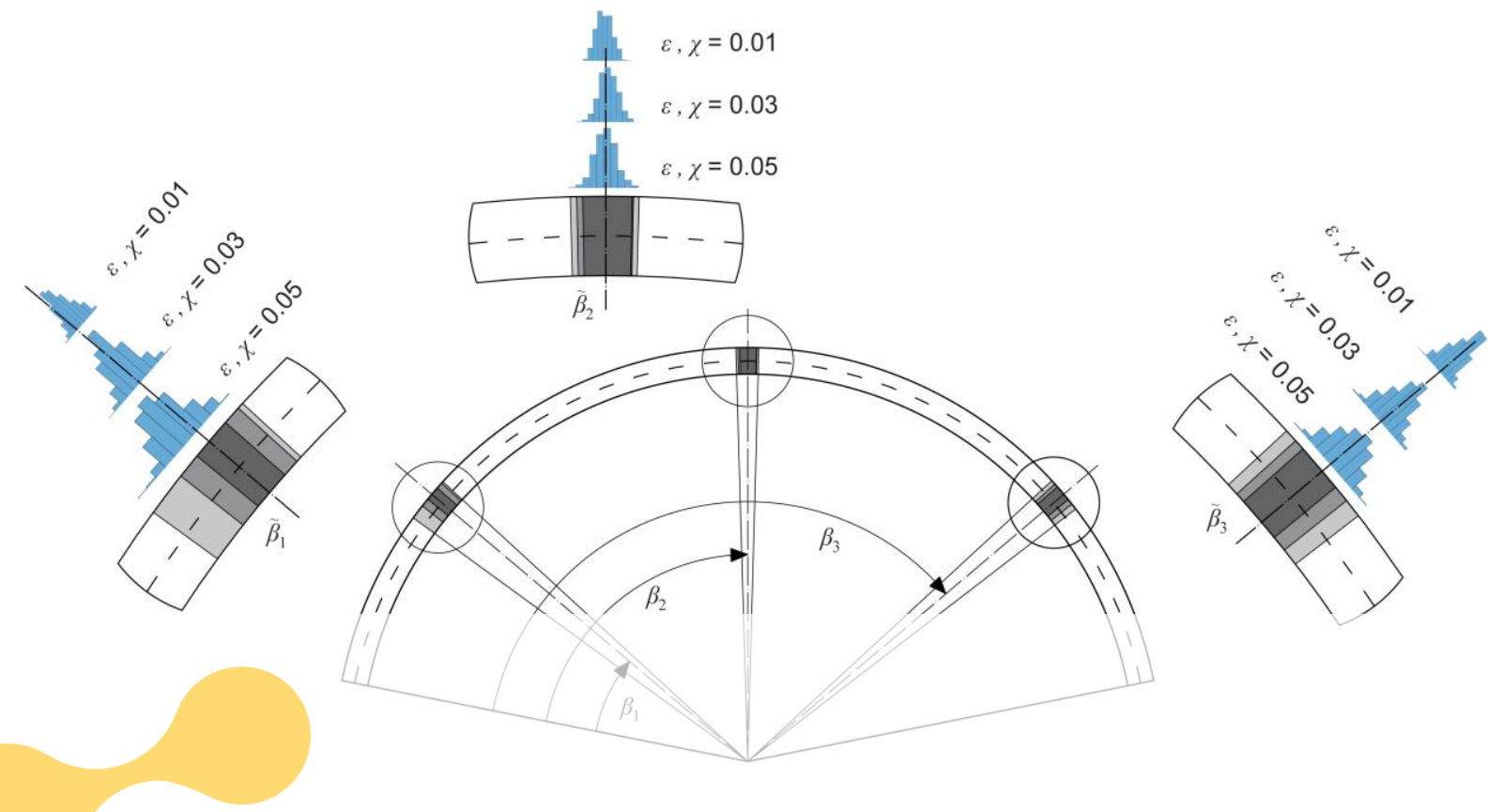

Figure 8: Probabilistic distributions of the hinges $\tilde{\beta}_{i}$, other than the fourth hinge occurring at one of the impost, identifying the collapse mechanism.

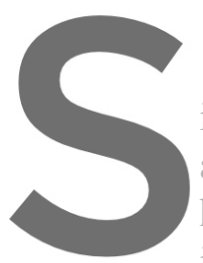

The results have irregularities the mean approach increase, up to about 3 highlight that also the identify the portion of the arch-ithich have a ntor
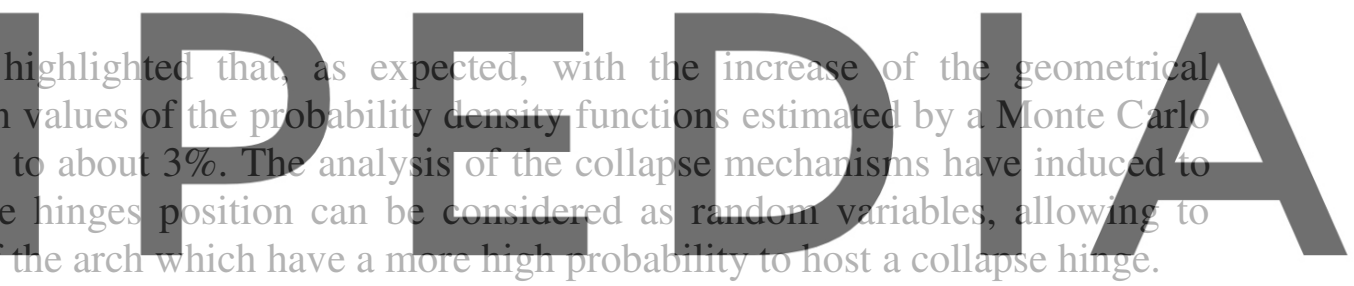

Register for free at https//www.scipedia.com to download the version without the watermark REFERENCES

[1] Giordano, E., Clementi, F., Nespeca, A. and Lenci, S. Damage assessment by numerical modeling of sant'agostino's sanctuary in offida during the central italy 2016-2017 seismic sequence. Frontiers in Built Environment (2019) 4(7):87.

[2] Cavalagli, N., Kita, A., Castaldo, V.L., Pisello, A.L. and Ubertini, F. Hierarchical environmental risk mapping of material degradation in historic masonry buildings: An integrated approach considering climate change and structural damage. Construction and Building Materials (2019) 215:998-1014.

[3] Grillanda, N., Chiozzi, A., Bondi, F., Tralli, A., Manconi, F., Stochino, F. and Cazzani, A. Numerical insights on the structural assessment of historical masonry stellar vaults: the case of Santa Maria del Monte in Cagliari. Continuum Mechanics and Thermodynamics (2019) in press, DOI: 10.1007/s00161-019-00752-8.

[4] Orlando, M., Betti, M. and Spinelli, P. Assessment of structural behaviour and seismic retrofitting for an Italian monumental masonry building. Journal of Building Engineering (2020) 29:101115.

[5] Cavalagli, N. and Gusella, V. Structural investigation of 18th-century ogival masonry 
domes: From Carlo Fontana to Bernardo Vittone. International Journal of Architectural Heritage (2015) 9(3):265-276.

[6] Galassi, S. and Tempesta, G. The Matlab code of the method based on the Full Range Factor for assessing the safety of masonry arches. MethodsX (2019) 6:1521-1542.

[7] Como, M., Di Carlo, F. and Coccia, S. Dynamic response of rocking cracked masonry walls. Meccanica (2019) 54(3):381-398.

[8] Brandonisio, G., Angelillo, M. and De Luca, A. Seismic capacity of buttressed masonry arches. Engineering Structures (2020) 215:110661.

[9] Nodargi, N.A. and Bisegna, P. Thrust line analysis revisited and applied to optimization of masonry arches. International Journal of Mechanical Sciences (2020) 179:105690.

[10] Cocchetti, G. and Rizzi, E. Static Upper/Lower Thrust and Kinematic Work Balance Stationarity for Least-Thickness Circular Masonry Arch Optimization. Journal of Optimization Theory and Applications (2020) 187(3):707-757.

[11] Fraddosio, A., Lepore, N. and Piccioni, M.D. Thrust Surface Method: An innovative approach for the three-dimensional lower bound Limit Analysis of masonry vaults. Engineering Structures (2020) 202:109846.

[12] Grillanda, N., Chiozzi, A., Milani, G. and Tralli, A. Efficient meta-heuristic mesh adaptation strategies for NURBS upper-bound limit analysis of curved three-dimensional masonry structures. Computers and Structures (2020) 236:106271.

[13] Bayraktar, A. and Hökelekli, E. Nonlinear soil deformability effects on the seismic damage mechanisms of brick and stone masonry arch bridges. International Journal of Damage Mechanics (2020) in press, DOI: 10.1177/1056789520974423.

[14] Zampieri, P. Horizontal capacity of single-span masonry bridges with intrados FRCM strengthening. Composite Structures (2020) 244:112238.

[15] Rovero, L., Galassi, S. and Misseri, G. Experimental and analytical investigation of bond behavior in glass fiber-reinforced composites based on gypsum and cement matrices. Composites Part B: Engineering (2020) 194:108051.

[16] Bayraktar, A. and Hökelekli, E. Seismic Performances of Different Spandrel Wall Strengthening Techniques in Masonry Arch Bridges. International Journal of Architectural Heritage (2020) in press, DOI: 10.1080/15583058.2020.1719234.

[17] Gusella, V. and Liberotti, R. Seismic Vulnerability of Sub-Structures: Vanvitelli's Modulus in Murena Palace. Buildings (2020) 10(9):164.

[18] Lourenço, P.B., Oliveira, D.V., Leite, J.C., Ingham J.M., Modena, C. and da Porto, F. Simplified indexes for the seismic assessment of masonry buildings: International database and validation. Engineering Failure Analysis (2013) 34:508-605.

[19] Tempesta, G. and Galassi S. Safety evaluation of masonry arches. a numerical procedure based on the thrust line closest to the geometrical axis. International Journal of Mechanical Sciences (2019) 155:206-221.

[20] Cazzani, A., Malagù, M. and Turco, E. Isogeometric analysis: a powerful numerical tool for the elastic analysis of historical masonry arches. Continuum Mechanics and Thermodynamics (2016) 28(1-2):139-156.

[21] Zampieri, P., Zanini, M., Faleschini, F. Seismic capacity of masonry arches with irregular abutments and arch thickness. Construction and Building Materials (2019) 201:786-806.

[22] Pepi, C., Cavalagli, N., Gusella, V., and Gioffrè, M. An integrated approach for the numerical modeling of severely damaged historic structures: Application to a masonry 
bridge. Advances in Engineering Software (2021) 151:102935.

[23] Cavalagli, N., Gusella, V. and Severini, L. The safety of masonry arches with uncertain geometry. Computers and Structures (2017) 188:17-31.

[24] Zampieri, P., Cavalagli, N., Gusella, V. and Pellegrino, C. Collapse displacements of masonry arch with geometrical uncertainties on spreading supports. Computers and Structures (2018) 208:118-129.

[25] Severini, L., Cavalagli, N., DeJong, M. and Gusella, V. Dynamic response of masonry arch with geometrical irregularities subjected to a pulse-type ground motion. Nonlinear Dynamics (2018) 91(1):609-624.

[26] Gusella, V. Geometrical uncertainty in mechanics and random curves in space. Probabilistic Engineering Mechanics (2020) 62:103102.

[27] Cavalagli, N., Gusella, V. and Liberotti, R. Effect of Shape Uncertainties on the Collapse Condition of the Circular Masonry Arch. In: B. Abali and I. Giorgio (Eds.): Developments and Novel Approaches in Nonlinear Solid Body Mechanics. Advanced Structured Materials, Springer, Cham 130 (2020), pp. 455-467.

[28] Cavalagli, N., Gusella, V. and Liberotti, R. The Role of Shape Irregularities on the Lateral Loads Bearing Capacity of Circular Masonry Arches. In: A. Carcaterra et al. (Eds.): Proceedings of XXIV AIMETA Conference 2019. AIMETA 2019. Lecture Notes in Mechanical Engineering, Springer Cham (2020), pp. 2069-2081.

[29] Heyman, J. (1969) The safety of masonry arches. International Journal of Mechanical Sciences (1969) 11(4):363-382. 